\title{
A RAPIDLY PROGRESSIVE CASE OF ANTI-NMDAR ENCEPHALITIS WITH PRIMARY PSYCHIATRIC SYMPTOMS
}

\author{
Buket Koparal ${ }^{1}$, Saba Çiçek ${ }^{2}$, Mustafa Ender Taner ${ }^{2}$ \& Aslı Kuruoğlu ${ }^{2}$ \\ ${ }^{l}$ Department of Psychiatry, Recep Tayyip Erdogan University Training and Research Hospital, Rize, Turkey \\ ${ }^{2}$ Department of Psychiatry, School of Medicine, Gazi University, Ankara, Turkey
}

received: 8.8.2020;

revised: 7.11.2020;

accepted: 22.11 .2020

$* * * * *$

\section{INTRODUCTION}

Since the introduction by Dalmau et al in 2007, numbers of reported anti-NMDAR encephalitis cases have increased with the growing interest of professionals in the diagnosis of this neurological condition (Dalmau et al. 2007). Although it was initially thought to be a rare phenomenon, more than 700 patients have been reported so far (Warren et al. 2018). The majority of the anti-NMDAR encephalitis cases are first seen by psychiatrists due to acute or subacute manifestations of psychiatric symptoms (Kayser \& Dalmau 2011a). Agitation, bizarre, and disinhibited behaviors, delusions, and hallucinations are the most commonly reported symptoms in these patients (Barry et al. 2015). Approximately $75 \%$ of the patients fully recover or have mild deficits in the long-term, while $25 \%$ remain severely disabled; and the anti-NMDAR encephalitis mortality rate is estimated to be around $4 \%$ (Warren et al. 2018). In this report, we aimed to present a rapidly progressing case of anti-NMDAR encephalitis with early psychiatric symptoms.

\section{CASE}

Female patient, aged 33, was admitted to our outpatient clinic by her family due to her "odd and blank" gazes, sudden, short crying spells, uneven temper, and emotional dullness which abruptly started 15 days ago. Before her referral to the clinic, she had already used fluoxetine $20 \mathrm{mg}$, lorazepam $2 \mathrm{mg}$, and quetiapine $200 \mathrm{mg}$ in different admissions for her symptoms. In each outpatient visit, those medications had been discontinued because none of them were beneficial. Despite the previous pharmacological interventions, her situation had progressed. She had told her husband she could communicate with God and would be punished for her sins; but for the last three days, she had refused to talk.

Her personal history revealed that she had to close down her business almost one year ago due to her debts; however, there were no psychiatric complaints back then. She had a cheerful, vibrant personality in the past; but her behaviors in the last few days were unexpected. She had no history of substance abuse.
She had recently been diagnosed with allergic eczema and had received antihistamines one month before her psychiatric admission. After her hospitalization for further investigation; she had intermittent agitations with catatonia and mutism and her speech was mostly incoherent. Despite these clinical features; her initial physical and neurological examination and laboratory workup was normal, so we decided to follow her up without any psychiatric medication. Her agitations were managed by occasional parenteral haloperidol injections as needed, but she eventually developed orofacial dyskinesia (sucking-like movements). Her electroencephalography (EEG) showed widespread delta, theta and triphasic wave patterns and magnetic resonance imaging (MRI) revealed asymmetric signal changes in the right temporal horn. On the fourth day of her follow-up, she had few myoclonic seizures and one status epilepticus activity which was managed by intravenous diazepam. On the following day, her vital signs became unstable; and she had rigidity, high fever, elevated CK levels, and mild leucocytosis which raised the suspicion of the neuroleptic malignant syndrome. Therefore she was started on bromocriptine treatment, but she was later transferred to the intensive care unit due to the continued deterioration of her medical condition. Lumbar puncture was performed and viral and anti-NMDAR encephalitis panels were studied. AntiNMDAR antibodies were found positive in both serum and cerebrospinal fluid (CSF). Methylprednisolone treatment was administered for 7 days and intravenous immunoglobulin (IVIG) treatment was planned. Plasmapheresis could not be performed due to her autonomic instability. Whole-body computerized tomography (CT) scan was done to scan for malignancy; and an abdominal CT scan showed a right adnexal lesion which was consistent with ovarian teratoma. The patient was later scheduled for tumor resection; however, she died upon the $12^{\text {th }}$ day of the ICU admission due to cardiovascular collapse.

\section{DISCUSSION}

Anti-NMDAR encephalitis is a neurological disease that has an acute or subacute onset and may present with emotional and behavioral changes, short- 
term memory problems, cognitive impairment, and seizures (Hermans et al. 2018). The typical course of the illness includes flu-like prodrome which is later followed by the neuropsychiatric phase including cognitive and behavioral symptoms. Delusions, hallucinations, mania, agitation, changes in speech, disorganized behavior, movement abnormalities, dysautonomia, hypoventilation, seizures, executive dysfunction, impulsivity, sleep abnormalities can be seen through the course of the illness (Kruse et al. 2015). Even though they are not pathognomonic; MRI may show hyperintensities in cortical or subcortical brain regions. EEG is usually abnormal with slow and disorganized activity in the delta/theta range (Kayser and Dalmau $2011 \mathrm{~b})$. The definitive diagnosis is made by detecting specific antibodies in serum or CSF (Graus et al. 2016). Almost half of the cases are associated with neoplasms with the majority being ovarian teratoma. Interestingly, psychiatric symptoms are found to be more common in teratoma related cases (Warren et al. 2018). The treatment includes a symptomatic approach and first-line (steroids, IVIG, plasma exchange, or both) or second-line (rituximab, cyclophosphamide, or other) immunotherapies; however, rituximab is increasingly being considered as first-line therapy (Graus et al. 2016). Early diagnosis, no history of ICU, initiation of immunotherapy, and tumor removal (if present) are considered as positive prognostic factors for antiNMDAR encephalitis (Hermans et al. 2018).

Reports on the pathophysiology of anti-NMDAR encephalitis suggest that flu-like symptoms in the prodromal stage of the disease may trigger an autoimmune reaction (Dalmau et al. 2007). Even though our patient had no flu-like prodromal stage; she was previously treated for dermatitis. It can be speculated that dermatitis may have also acted as another autoimmune trigger, but this assumption needs more clinical evidence. The patients with anti-NMDAR encephalitis may have many psychiatric diagnoses in the process until they get a definitive neurological diagnosis. Schizophrenia diagnosis among these patients is very frequent, due to their psychotic symptoms (Kayser \& Dalmau 2011a). There are reports of some cases who were treated with psychosis diagnosis for several years (Huang et al. 2015, Simabukuro et al. 2015). In our case, psychiatric complaints were the main reason for the admission; yet the patient had a poor response to initial and previous psychiatric treatment modalities. Not including the previous psychiatric treatments, the time spent until the definitive diagnosis after her admission was 33 days. This delay in diagnosis of our patient can be linked to the symptoms mimicking a primary psychiatric illness; lack of neurological findings at the admission; the presence of a psychosocial stressor and the rapid clinical progression. This similarity of the symptoms between psychosis and encephalitis has given rise to the concept of autoimmune psychosis. Detection of anti-NMDAR antibodies may lead to new trends for treating psychosis.
In this case report, we wanted to draw attention to a rare neurological disorder which primarily presents with psychiatric symptoms and raise medical awareness among clinicians in order to provide an early and effective diagnosis and treatment potential. Clinicians should consider anti-NMDAR encephalitis as a possible diagnosis especially in patients with no familial or personal psychiatric history, acute presentation, complex neuro-psychiatric symptoms, and poor response to psychiatric medications. Although we can not fully clarify the mechanisms caused by the auto antibodies underlying all the psychotic symptomatology; the fact that auto-immunity plays a role in some patients may facilitate the discovery of new approaches for the treatment.

\section{Acknowledgments: None.}

\section{Conflict of interest: None to declare.}

\section{Contribution of individual authors:}

Buket Koparal: writing the paper and search for literature.

Saba Çiçek: writing the paper.

Mustafa Ender Taner \& Aslı Kuruoğlu: critical revision of manuscript.

\section{References}

1. Barry H, Byrne S, Barrett E, Murphy KC, Cotter DR: Anti-N-methyl-d-aspartate receptor encephalitis: review of clinical presentation, diagnosis and treatment. BJ Psych Bull 2015; 39:19-23

2. Dalmau J, Tuzun E, Wu HY, Masjuan J, Rossi JE, Voloschin A, Baehring JM, Shimazaki H, Koide R, King D, Mason W, Sansing LH, Dichter MA, Rosenfeld MR, Lynch DR: Paraneoplastic anti-N-methyl-D-aspartate receptor encephalitis associated with ovarian teratoma. Ann Neurol 2007; 61:25-36

3. Graus F, Titulaer MJ, Balu R, Benseler $S$, Bien $C G$, Cellucci T, Cortese I, Dale RC, Gelfand JM, Geschwind $M$, Glaser CA, Honnorat J, Hoftberger R, Iizuka T, Irani SR, Lancaster E, Leypoldt F, Pruss H, Rae-Grant A, Reindl M, Rosenfeld MR, Rostasy K, Saiz A, Venkatesan $A$, Vincent A, Wandinger KP, Waters P, Dalmau J: A clinical approach to diagnosis of autoimmune encephalitis. Lancet Neurol 2016; 15:391-404

4. Hermans T, Santens P, Matton C, Oostra K, Heylens $G$, Herremans S, Lemmens GMD: Anti-NMDA receptor encephalitis: still unknown and underdiagnosed by physicians and especially by psychiatrists? Acta Clin Belg 2018; 73:364-367

5. Huang $C$, Kang $Y$, Zhang B, Li B, Qiu C, Liu S, Ren H, Yang $Y$, Liu X, Li T, Guo W: Anti-N-methyl-d-aspartate receptor encephalitis in a patient with a 7-year history of being diagnosed as schizophrenia: complexities in diagnosis and treatment. Neuropsychiatr Dis Treat 2015; 11:1437-1442

6. Kayser MS, Dalmau J: Anti-NMDA Receptor Encephalitis in Psychiatry. Curr Psychiatry Rev 2011a; 7:189-193 
7. Kayser MS, Dalmau J: The emerging link between autoimmune disorders and neuropsychiatric disease. J Neuropsychiatry Clin Neurosci 2011b; 23:90-97

8. Kruse JL, Lapid MI, Lennon VA, Klein CJ, Toole OO, Pittock SJ, Strand EA, Frye MA, McKeon A: Psychiatric Autoimmunity: N-Methyl-D-Aspartate Receptor $\operatorname{Ig} G$ and Beyond. Psychosomatics 2015; 56:227-241

9. Simabukuro MM, Freitas CHA, Castro LHM: A patient with a long history of relapsing psychosis and mania presenting with anti-NMDA receptor encephalitis ten years after first episode. Dement Neuropsychol 2015; 9:311-314

10. Warren $N$, Siskind D, O'Gorman $C$ : Refining the psychiatric syndrome of anti- $N$-methyl-d-aspartate receptor encephalitis. Acta Psychiatr Scand 2018; 138:401-408

Correspondence:

Buket Koparal, MD

Department of Psychiatry, Recep Tayyip Erdogan University Training and Research Hospital Rize, Turkey

E-mail:bkt_svnc@hotmail.com 